doi: http://dx.doi.org/10.7124/bc.0009A5

\section{A description and review of a new mitotic spindle component, "tethers", elastic mitotic structures that extend between telomeres throughout anaphase'}

\section{Arthur Forer \\ Biology Department, York University, Toronto, ON Canada M3J 1P3 \\ aforer@yorku.ca}

Tethers extend between the separating telomeres of anaphase chromosomes in all animal meiotic and mitotic cells tested so far: flatworms, insects, spiders, marsupials and humans [Forer et al., European Journal of Cell Biology 96 (2017) 504-514]. They are identified experimentally: when a laser microbeam severs an anaphase chromosome's arm, the resultant arm fragment rapidly moves across the equator to the partner telomere. Telomeres are required for arm-fragment movements: arm fragments stop moving when either telomere is ablated, and when an arm fragment is severed only the telomere-containing piece moves. Arm-fragment movements are not due to microtubules: taxol abolishes spindle microtubule transport properties yet arm fragments still move at normal speeds. Arm-fragment movements are not due to ultra-fine DNA strands: only a few chromosomes in each cell are connected by those strands whereas all chromosomes have tethers, and those strands slow anaphase chromosome movements whereas tethers do not. Thus elastic tethers that extend between the telomeres of separating anaphase chromosomes cause movements of arm frag- ments. We know some properties of tethers. They connect all separating chromosomes, but in meiosis they connect only 2 of the 4 separating chromatids. As the distance between separating telomeres increases, the arm fragments move slower and slower, do not move as far, and at longer tether lengths do not move. The slowing and absence of arm-fragment movements is due to inelasticity and not detachment of tethers: tethers stretch the chromosomes they are connected with because when tethers are cut with a laser both chromosome arms shrink by about $10 \%$, at all stages of anaphase and telophase, even when arm fragments do not move. We lack important information about tethers. We do not know what they are made of, though immunofluorescence staining has identified the giant elastic protein titin extending between the telomeres of separating anaphase chromosomes. We do not know why tethers become inelastic, but this might be due to dephosphorylation: a phosphatase inhibitor added during early anaphase causes the tethers to maintain their elasticity throughout telophase instead of becoming inelastic at later stages. We do not know the general function of tethers, but in crane-fly spermatocytes tethers regulate speeds of separating chromosomes: movements of separating half-bivalents in crane-fly meiosis are coupled (e.g., ultraviolet microbeam irradiation of a spindle fibre causes the attached half-bivalent to stop moving as well as the partner heading to the other pole), but the movements become uncoupled if one severs the tethers before irradiating the spindle fibre. Thus the coupling of movements requires tethers. We do not know if tethers are present in spindles in plant cells. 